\title{
Solutions to open problems in Neeb's recent survey on infinite-dimensional Lie groups
}

\author{
Helge Glöckner
}

\begin{abstract}
We solve three open problems concerning infinite-dimensional Lie groups posed in a recent survey article by K.-H. Neeb [21. Moreover, we prove a result by the author announced in [21], which answers a question posed in an earlier, unpublished version of the survey.

Classification: 22E65 (Primary) 58B10 (Secondary)

Key words: infinite-dimensional Lie group, open problem, initial submanifold, initial Lie subgroup, direct limit group, topological group with Lie algebra, Borel's Theorem, Taylor series, diffeomorphism
\end{abstract}

\section{Introduction}

The recent article 21] by K.-H. Neeb surveyed the state of the art of the theory of infinite-dimensional Lie groups, and described typical open problems. In this article, we provide full solutions to three of these:

- We describe a subgroup of an infinite-dimensional Lie group which is not an initial Lie subgroup (Section 11). This answers [21, Problem II.6], the "Initial Subgroup Problem," in the negative.

- We show that every subgroup of a direct $\operatorname{limit} G=\lim G_{n}$ of finitedimensional Lie groups is an initial Lie subgroup (Section 2). This answers [21, Problem VII.3] in the affirmative.

- We show that every direct $\operatorname{limit} G=\lim G_{n}$ of finite-dimensional Lie groups is a topological group with Lie algebra in the sense of [16] (Section 3). This answers [21, Problem VII.2] in the affirmative.

* Supported by the German Research Foundation (DFG), projects GL 357/5-1 and GL $357 / 7-1$ 
We also prove a result by the author announced in [21, Proposition VI.2.11]: Let $M$ be $\sigma$-compact finite-dimensional smooth manifold of dimension $n>0$, and $\operatorname{Diff}_{c}(M)$ be the Lie group of smooth diffeomorphisms $\gamma: M \rightarrow M$ which are compactly supported in the sense that the closure of $\{x \in M: \gamma(x) \neq x\}$ is compact (see [18] and [9]). Given $p \in M$, let $\operatorname{Diff}_{c}(M)_{p}$ be its stabilizer, and $\operatorname{Diff}_{c}(M)_{p, 0}$ be the subgroup of all $\gamma \in \operatorname{Diff}_{c}(M)_{p}$ such that $\operatorname{det} T_{p}(\gamma)>0$, where $T_{p}(\gamma): T_{p} M \rightarrow T_{p} M$ is the tangent map. Let $\mathrm{Gf}_{n}(\mathbb{R}) \subseteq \mathbb{R} \llbracket x_{1}, \ldots, x_{n} \rrbracket$ be the group of all formal diffeomorphisms of $\mathbb{R}^{n}$, and $\mathrm{Gf}_{n}(\mathbb{R})_{0}$ be the subgroup of all formal diffeomorphisms whose linear part has positive determinant (see [21, pp. 426-427] and [23]). In Section 4, we establish a version of É. Borel's theorem on the existence of smooth maps with a given Taylor series:

Borel's Theorem for Diffeomorphisms. Let a finite-dimensional smooth manifold $M$ of dimension $n$ be given, $p \in M$ and $\phi$ be a local chart around $p$ such that $\phi(p)=0$. For each $\gamma \in \operatorname{Diff}_{c}(M)$, let $T_{p}^{\infty}(\gamma)$ denote the Taylor series of $\phi \circ \gamma \circ \phi^{-1}$ around 0 . Then

$$
T_{p}^{\infty}: \operatorname{Diff}_{c}(M)_{p, 0} \rightarrow \operatorname{Gf}_{n}(\mathbb{R})_{0}
$$

is a surjective homomorphism of Lie groups.

We mention that further problems from [21] have an affirmative answer:

In [3], it is shown that the group $\mathrm{Gh}_{n}(\mathbb{C})$ of germs of analytic diffeomorphisms around $0 \in \mathbb{C}^{n}$ (as first considered in [22]) is a regular Lie group in Milnor's sense 1 This answers the first half of [21, Problem VI.5] in the affirmative.

Using a version of the Frobenius Theorem for co-Banach distributions on infinite-dimensional manifolds 2 one obtains the following result concerning integral subgroups in the sense of [21, Definition IV.4.7] (see [11]):

Let $G$ be a Lie group modelled on a locally convex space and $\mathfrak{h} \subseteq L(G)$ be a Lie subalgebra such that $L(G)=\mathfrak{h} \oplus X$ for some Banach space $X \subseteq L(G)$. Then there exists an integral subgroup $H \subseteq G$ such that $L(H)=\mathfrak{h}$.

Note that a complement $X$ always exists if $\mathfrak{h}$ has finite codimension. Hence [21, Problem VI.4] has an affirmative answer (even if $G$ is not regular).

Finally, we mention that the idea expressed in [21, Problem VII.5] has been taken up in [8, Lemma 14.5 and Section 15].

\footnotetext{
${ }^{1}$ Likewise for groups of germs of diffeomorphisms around a compact set, as in 8 .

${ }^{2}$ The adaptation to Keller's $C_{c}^{\infty}$-theory (our setting of infinite-dimensional calculus) of Hiltunen's Frobenius Theorem from [13] (to be found in [11]).
} 
Prerequisites. The reader is referred to [21], [12] or [4] for the setting of infinite-dimensional Lie groups, manifolds and $C^{k}$-mappings used in this article (cf. [19] for the case of sequentially complete modelling spaces). The words "Lie group" and "manifold" will always refer to Lie groups and manifolds modelled on locally convex spaces.

\section{Example of a subgroup of a Lie group which is not an initial Lie subgroup}

We shall use the following terminology.

Definition 1.1 Let $\mathbb{N}:=\{1,2, \ldots\}, k \in \mathbb{N} \cup\{\infty\}$ and $M$ be a $C^{k}$-manifold. A subset $N \subseteq M$ is called a $C^{k}$-initial submanifold of $M$ if $N$ can be made a $C^{k}$-manifold with the following properties:

(a) The inclusion map $\lambda: N \rightarrow M$ is a $C^{k}$-map; and

(b) If $X$ is a $C^{k}$-manifold and $f: X \rightarrow N$ a map such that $\lambda \circ f$ is $C^{k}$, then $f$ is $C^{k}$.

Such a manifold structure is called $C^{k}$-initial in $M$. If $G$ is a Lie group, let $L(G):=T_{1}(G)$ be its Lie algebra. Following [21, Definition II.6.1], we say that a subgroup $H \subseteq G$ is an initial Lie subgroup if it can be given a smooth manifold structure which makes $H$ a Lie group, is $C^{k}$-initial in $G$ for each $k \in \mathbb{N} \cup\{\infty\}$, and gives rise to an injective tangent map $L(\lambda):=$ $T_{1}(\lambda): L(H) \rightarrow L(G)$.

Remark 1.2 It means additional information if $N$ and $X$ may even be $C^{k}$-manifolds with $C^{k}$-boundary in Definition 1.1, or more generally $C^{k}$ manifolds "with rough boundary" as in [12] We then call $N$ a strongly initial $C^{k}$-submanifold of $M$. Notably, the strengthened concept enables maps $f: X \rightarrow N$ to be considered if $X=[0,1], X=[0,1]^{2}$ or $X$ is a simplex.

Let $\mathbb{R}^{\mathbb{N}}$ be the Fréchet space of real sequences (with the product topology). We show:

\footnotetext{
${ }^{3}$ The latter manifolds locally look like a locally convex subset with dense interior in the modelling space. They include manifolds with $C^{k}$-boundary and manifolds with corners as special cases.
} 
Theorem 1.3 For each $k \in \mathbb{N} \cup\{\infty\}$, the additive subgroup $N:=\ell^{\infty}$ of bounded sequences is not a $C^{k}$-initial submanifold of $M:=\mathbb{R}^{\mathbb{N}}$. In particular, it is not an initial Lie subgroup.

Proof. We shall use a well-known fact: A map from a $C^{k}$-manifold to $\mathbb{R}^{\mathbb{N}}$ (or any direct product of locally convex spaces) is $C^{k}$ if and only if all of its components are $C^{k}$ (see [12, Chapter 1] or [1, Lemma 10.2]).

It is useful to note first that, for each $m \in \mathbb{N}$, the topological product $K_{m}:=[-m, m]^{\mathbb{N}}$ is a metrizable compact topological space (and therefore separable), whence every open subset $U \subseteq K_{m}$ is $\sigma$-compact.

If the theorem was false, we could equip $N$ with a $C^{k}$-initial manifold structure. Assuming this, write $\lambda: N \rightarrow M$ for the inclusion map (which is $C^{k}$ ). Given $m \in \mathbb{N}$, choose an injective $C^{k}$-map $\left.h_{m}: \mathbb{R} \rightarrow\right]-2 m, 2 m[$ such that $h_{m}([-1,1])=[-m, m]$. Then the injective map

$$
g_{m}: \mathbb{R}^{\mathbb{N}} \rightarrow N, \quad\left(x_{n}\right)_{n \in \mathbb{N}} \mapsto\left(h_{m}\left(x_{n}\right)\right)_{n \in \mathbb{N}}
$$

is $C^{k}$ by initiality, exploiting that $\lambda \circ g_{m}$ is $C^{k}$ since its components are $C^{k}$. In particular, $g_{m}$ is continuous, entailing that

$$
\left.g_{m}\right|_{[-1,1]^{\mathbb{N}}}:[-1,1]^{\mathbb{N}} \rightarrow N
$$

is a topological embedding. Since $g_{m}\left([-1,1]^{\mathbb{N}}\right)=K_{m}$ as a set and $\lambda \circ g_{m}$ takes $[-1,1]^{\mathbb{N}}$ homeomorphically onto $K_{m}$, it follows that $N$ induces the given compact topology on $K_{m}$.

By the preceding, $N=\bigcup_{m \in \mathbb{N}} K_{m}$ is $\sigma$-compact. Let $E$ be the modelling space of $N$ and $\phi: N \supseteq U \rightarrow V \subseteq E$ be a chart of $N$, defined on a non-empty open set $U \subseteq N$. Then $U=\bigcup_{m \in \mathbb{N}}\left(U \cap K_{m}\right)$ where $U \cap K_{m}$ is $\sigma$-compact for each $m \in \mathbb{N}$ (as recalled above), and hence $U$ is $\sigma$-compact. Consequently, also the locally convex space $E$ is $\sigma$-compact, because it contains a $\sigma$-compact set with non-empty interior.

If $x \in N$, then $T_{x}(N) \cong E$ is $\sigma$-compact. We claim that the tangent map $T_{x}(\lambda): T_{x}(N) \rightarrow T_{x}(M)$ is surjective.

If this is true, then $\mathbb{R}^{\mathbb{N}} \cong T_{x}(M)=T_{x}(\lambda)\left(T_{x}(N)\right)$ is $\sigma$-compact. But this is absurd (by a Baire argument), and the theorem is proved by contradiction. To prove the claim, identify $T_{x}(M)$ with $\mathbb{R}^{\mathbb{N}}$. If $y=\left(y_{n}\right)_{n \in \mathbb{N}} \in \mathbb{R}^{\mathbb{N}}$, then

$$
g: \mathbb{R} \rightarrow N, \quad g(t):=\left(x_{n}+\sin \left(t y_{n}\right)\right)_{n \in \mathbb{N}}
$$


is a function such that $\lambda \circ g: \mathbb{R} \rightarrow \mathbb{R}^{\mathbb{N}}$ is $C^{k}$, because all components of this composition are $C^{k}$. Furthermore, $T_{x}(\lambda)\left(g^{\prime}(0)\right)=(\lambda \circ g)^{\prime}(0)=y$.

Our proof shows that Theorem 1.3 remains valid if, instead of our general setting, only manifolds modelled on complete (or sequentially complete, or Mackey complete) locally convex spaces are considered. Also, it remains valid if manifolds are replaced by manifolds with rough boundary.

\section{Subgroups of direct limit groups are initial}

If $G_{1} \subseteq G_{2} \subseteq \cdots$ is a sequence of finite-dimensional real Lie groups such that the inclusion maps $G_{n} \rightarrow G_{n+1}$ are smooth homomorphisms, then $G:=$ $\bigcup_{n \in \mathbb{N}} G_{n}$ admits a Lie group structure making each inclusion map $G_{n} \rightarrow G$ a smooth homomorphism, and such that $G=\lim G_{n}$ in the category of smooth Lie groups modelled on locally convex spaces [7, Theorem 4.3] (cf. [20], [17, Theorem 47.9] and [5] for special cases). Then $G=\lim G_{n}$ also as a topological space, and as a $C^{k}$-manifold, for each $k \in \mathbb{N}_{0} \cup\{\infty\}$. Furthermore,

$$
L(G)=\bigcup_{n \in \mathbb{N}} L\left(G_{n}\right)=\lim _{\longrightarrow} L\left(G_{n}\right)
$$

as a locally convex space, topological space and $C^{k}$-manifold (see [7. Theorems 4.3 (a) and 3.1] for all of this). For brevity, we shall refer to $G=\lim _{\longrightarrow} G_{n}$ as a direct limit Lie group. In this section, we show:

Theorem 2.1 Let $G_{1} \subseteq G_{2} \subseteq \cdots$ be finite-dimensional Lie groups, such that the inclusion map $G_{n} \rightarrow G_{n+1}$ is a smooth homomorphism for each $n \in \mathbb{N}$. Let $G=\lim G_{n}$ be the direct limit Lie group, and $H \subseteq G$ be a subgroup. Equip $H_{n}:=G_{n} \cap H$ with the finite-dimensional real Lie group structure induced by $G_{n}$ (as in [2, Chapter III, §4.5, Proposition 9]) and $H$ with the Lie group structure making it the direct limit Lie group $H=\lim H_{n}$ (as in [7, Proposition 7.2]). Then the manifold structure on $H$ is strongly $C^{k}$-initial in $G$, for all $k \in \mathbb{N} \cup\{\infty\}$. Thus $H$ is an initial Lie subgroup of $G$.

The final conclusion is possible because the inclusion map $\lambda: H \rightarrow G$ gives rise to an embedding $L(\lambda): L(H) \rightarrow L(G)$ by [7, Proposition 7.2].

It is useful to recall some simple facts. 
2.2 The identity component of a direct limit Lie group $G=\underset{\longrightarrow}{\lim } G_{n}$ is

$$
G_{0}=\bigcup_{n \in \mathbb{N}}\left(G_{n}\right)_{0}=\lim _{\longrightarrow}\left(G_{n}\right)_{0}
$$

In particular, $G_{0}$ is a direct limit group as well.

2.3 Consider an ascending sequence $X_{1} \subseteq X_{2} \subseteq \cdots$ of topological spaces. Then $X:=\bigcup_{n \in \mathbb{N}} X_{n}$ is its direct limit in the category of topological spaces, if we declare $U \subseteq X$ open if and only if $U \cap X_{n}$ is open in $X_{n}$ for each $n \in \mathbb{N}$. The topology so obtained is called the direct limit topology. If $U_{n} \subseteq X_{n}$ are open subsets such that $U_{1} \subseteq U_{2} \subseteq \cdots$, then $U:=\bigcup_{n \in \mathbb{N}} U_{n}$ is open in $X$, because $U \cap X_{n}=\bigcup_{m \geq n} U_{m} \cap X_{n}$ is open in $X_{n}$ for each $n$.

Also the following lemma will be useful for the proof of Theorem 2.1 .

Lemma 2.4 Let $X$ be a finite-dimensional real vector space, $K \subseteq X$ be a non-empty compact set, $k \in \mathbb{N} \cup\{\infty\}, M$ be a finite-dimensional $C^{k}$-manifold and $f: U \rightarrow M$ be a $C^{k}$-map on an open neighbourhood $U$ of $K$ in $X$ such that $\left.f\right|_{K}$ is injective and the tangent map $T_{x}(f): T_{x}(X) \rightarrow T_{f(x)}(M)$ is invertible for each $x \in K$. Then there is an open neighbourhood $V \subseteq U$ of $K$ such that $f(V)$ is open in $M$ and $\left.f\right|_{V}$ is a $C^{k}$-diffeomorphism onto $f(V)$.

Proof. The proof of [8, Lemma 15.6] (where $M=X$ ) carries over.4

Proof of Theorem 2.1. We may assume that $G_{1}=H_{1}=\{1\}$. Let $X$ be a $C^{k}$-manifold (possibly with rough boundary) and $f: X \rightarrow H$ be a map such that $\lambda \circ f: X \rightarrow G$ is $C^{k}$, where $\lambda: H \rightarrow G$ is the inclusion map. Given $p \in X$, let $Y$ be the connected component of $X$ which contains $p$. Since $Y$ is open in $X$, it suffices to show that $\left.f(p)^{-1} f\right|_{Y}$ is $C^{k}$ on some open neighbourhood of $p$. After replacing $X$ with $Y$ and $f$ with $\left.f(p)^{-1} f\right|_{Y}$, we may assume that $X$ is connected and $f(p)=1$. Then $f(X)$ is contained in the identity component $G_{0}$ of $G$. Since $G_{0}=\lim \left(G_{n}\right)_{0}$ (see 2.2), after replacing $G$ with $G_{0}, G_{n}$ with $\left(G_{n}\right)_{0}$ and $H$ with $\vec{H} \cap G_{0}$, we may assume that $G$ and each $G_{n}$ is connected. Then $G_{n}$ is the integral subgroup of $G_{n+1}$ with Lie algebra $L\left(G_{n}\right)$. Since the latter is $C^{\infty}$-initial in $G_{n+1}$, it follows that

$$
L\left(G_{n}\right)=\left\{x \in L\left(G_{n+1}\right): \exp _{G_{n+1}}(\mathbb{R} x) \subseteq G_{n}\right\}
$$

\footnotetext{
${ }^{4}$ Unfortunately, there is a misprint: " $E$ " reads " $X$ " in the lemma.
} 
(see [21, Theorem IV.4.14]). We recall that

$$
L\left(H_{n}\right)=\left\{\gamma^{\prime}(0): \gamma \in C^{1}\left([0,1], G_{n}\right) \text { with } \gamma(0)=1 \text { and } \gamma([0,1]) \subseteq H_{n}\right\}
$$

and also

$$
L\left(H_{n}\right)=\left\{x \in L\left(G_{n}\right): \exp _{G_{n}}(\mathbb{R} x) \subseteq H_{n}\right\},
$$

(cf. [2, Chapter 3, §4.5] or [21, Theorem IV.4.14]). Combining (3) with (11) and the fact that $L(G)=\bigcup_{n \in \mathbb{N}} L\left(G_{n}\right)$, we see that

$$
L\left(H_{n}\right)=\left\{x \in L(G): \exp _{G}(\mathbb{R} x) \subseteq H_{n}\right\}
$$

and thus

$$
L(H) \cap L\left(G_{n}\right)=L\left(H_{n}\right) .
$$

Recursively, we find vector subspaces $E_{n} \subseteq L\left(G_{n}\right)$ for $n \in \mathbb{N}$ such that $E_{n-1} \subseteq E_{n}$ (if $n \geq 2$ ) and

$$
L\left(G_{n}\right)=L\left(H_{n}\right) \oplus E_{n} .
$$

In fact, if $n=1$ we choose $E_{1}:=\{0\}$. If $E_{1}, \ldots, E_{n}$ have been chosen, then

$$
L\left(H_{n+1}\right) \cap E_{n}=L\left(H_{n+1}\right) \cap L\left(G_{n}\right) \cap E_{n}=L\left(H_{n}\right) \cap E_{n}=\{0\},
$$

using (44) for the penultimate equality. Hence $E_{n}$ can be extended to a vector complement $E_{n+1}$ to $L\left(H_{n+1}\right)$ in $L\left(G_{n+1}\right)$.

Give $E:=\bigcup_{n \in \mathbb{N}} E_{n}=\lim _{n} E_{n}$ the locally convex direct limit topology. Then

$$
L(G)=\lim _{\longrightarrow}\left(L\left(H_{n}\right) \oplus E_{n}\right)=\lim _{\longrightarrow} L\left(H_{n}\right) \oplus \underset{\longrightarrow}{\lim } E_{n}=L(H) \oplus E
$$

as a locally convex space (see, e.g., [14, Theorem 3.4]). For each $n \in \mathbb{N}$, let $R_{n} \subseteq L\left(H_{n+1}\right)$ and $S_{n} \subseteq E_{n+1}$ be vector subspaces such that

$$
L\left(H_{n+1}\right)=L\left(H_{n}\right) \oplus R_{n} \text { and } \quad E_{n+1}=E_{n} \oplus S_{n} .
$$

We now pick a basis $\mathcal{B}$ of $L(G)$ such that $\mathcal{B} \cap L\left(H_{n}\right)$ is a basis of $L\left(H_{n}\right)$, $\mathcal{B} \cap E_{n}$ is a basis of $E_{n}, \mathcal{B} \cap R_{n}$ is a basis of $R_{n}$ and $\mathcal{B} \cap S_{n}$ is a basis of $S_{n}$, for each $n \in \mathbb{N}$. It can be used to introduce the supremum norm

$$
\|.\|: L(G) \rightarrow\left[0, \infty\left[, \quad\left\|\sum_{b \in \mathcal{B}} t_{b} b\right\|:=\sup _{b \in \mathcal{B}}\left|t_{b}\right| \quad \text { for }\left(t_{b}\right)_{b \in \mathcal{B}} \in \mathbb{R}^{(\mathcal{B})} .\right.\right.
$$


Given $t>0$ and $n \in \mathbb{N}$, abbreviate

$$
\begin{aligned}
& U_{t}^{n}:=\left\{x \in L\left(H_{n}\right):\|x\|<t\right\}, \quad V_{t}^{n}:=\left\{x \in E_{n}:\|x\|<t\right\}, \\
& X_{t}^{n}:=\left\{x \in R_{n}:\|x\|<t\right\}, \quad \text { and } \quad Y_{t}^{n}:=\left\{x \in S_{n}:\|x\|<t\right\} .
\end{aligned}
$$

Then $U_{t}^{n+1}=U_{t}^{n} \times X_{t}^{n}$ and $V_{t}^{n+1}=V_{t}^{n} \times Y_{t}^{n}$. Let $2=t_{1}>t_{2}>\cdots>1$.

Claim. There are $C^{\infty}{ }_{-m a p s} \eta_{n}: V_{t_{n}}^{n} \rightarrow G_{n}$ for $n \in \mathbb{N}$ and $C^{\infty}$-diffeomorphisms $\gamma_{n}: U_{t_{n}}^{n} \rightarrow H_{n}$ onto open identity neighbourhoods in $H_{n}$, such that $\gamma_{n}(0)=$ $\eta_{n}(0)=1$,

$$
g_{n}: U_{t_{n}}^{n} \times V_{t_{n}}^{n} \rightarrow G_{n}, \quad g_{n}(x, y):=\gamma_{n}(x) \eta_{n}(y)
$$

is a $C^{\infty}$-diffeomorphism onto an open identity neighbourhood in $G_{n}$, and

$$
\left.\gamma_{k}\right|_{U_{t_{k}}^{\ell}}=\left.\gamma_{\ell}\right|_{U_{t_{k}}^{\ell}} \text { as well as }\left.\quad \eta_{k}\right|_{V_{t_{k}}^{\ell}}=\left.\gamma_{\ell}\right|_{V_{t_{k}}^{\ell}} \quad \text { for all } 1 \leq \ell \leq k \leq n \text {. }
$$

If this claim is true, then $\sigma_{n}:=\left.\gamma_{n}\right|_{U_{1}^{n}}$ is a $C^{\infty}$-diffeomorphism onto the open identity neighbourhood $Q_{n}:=\gamma_{n}\left(U_{1}^{n}\right)$ in $H_{n}$, the mapping $h_{n}:=\left.g_{n}\right|_{U_{1}^{n} \times V_{1}^{n}}$ is a $C^{\infty}$-diffeomorphism onto the open identity neighbourhood $g_{n}\left(U_{1}^{n} \times V_{1}^{n}\right)$ $=: P_{n}$ in $G_{n}$, and $\tau_{n}:=\left.\eta_{n}\right|_{V_{1}^{n}}: V_{1}^{n} \rightarrow G_{n}$ is a smooth map (and in fact an immersion and topological embedding, since $h_{n}(x, y)=\sigma(x) \tau(y)$ and $h_{n}$ is a diffeomorphism.). Then $U:=\bigcup_{n \in \mathbb{N}} U_{1}^{n}$ is an open 0-neighbourhood in $L(H)$ and $Q:=\bigcup_{n \in \mathbb{N}} Q_{n}$ is a connected, open identity neighbourhood in $H$ (see 2.3). Moreover,

$$
\sigma:=\lim _{\longrightarrow} \sigma_{n}: L(H) \supseteq U \rightarrow Q \subseteq H
$$

is a $C^{\infty}$-diffeomorphism (cf. [7, Lemma 1.9 and Proposition 3.3]). Also, $W:=\bigcup_{n \in \mathbb{N}}\left(U_{1}^{n} \times V_{1}^{n}\right)$ is an open 0-neighbourhood in $L(G), P:=\bigcup_{n \in \mathbb{N}} P_{n}$ is an open identity neighbourhood in $G$, and

$$
h:=\lim _{\longrightarrow} h_{n}: L(G) \supseteq W \rightarrow P \subseteq G
$$

is a $C^{\infty}$-diffeomorphism. Finally, $V:=\bigcup_{n \in \mathbb{N}} V_{n}$ is an open 0-neighbourhood in $E$ and

$$
\tau:=\lim _{\longrightarrow} \tau_{n}: E \supseteq V \rightarrow G
$$

is a $C^{\infty}$-map (and actually an immersion and topological embedding, since $h(x, y)=\sigma(x) \tau(y)$ and $h$ is a diffeomorphism). There exists a connected open neighbourhood $Z \subseteq X$ of $p$ such that $f(Z) \subseteq P$. We now show that

$$
f(Z) \subseteq Q
$$


To this end, let $q \in Z$. There exists a $C^{1}$-map $\theta:[0,1] \rightarrow Z$ such that $\theta(0)=p$ and $\theta(1)=q$. By [7, Lemma $1.7(\mathrm{~d})]$, there exists $n \in \mathbb{N}$ such that

$$
f(\theta([0,1])) \subseteq G_{n} .
$$

Then the left logarithmic derivative $\delta(f \circ \theta):[0,1] \rightarrow L(G)$ is a continuous map taking its values in $L\left(H_{n}\right)$ (by (2) and [2, Chapter 3, §4.5, Lemma 4]) 5 As a consequence of the standard Existence and Uniqueness Theorem for solutions to ordinary differential equations, there exists a unique $C^{1}$-curve $\kappa:[0,1] \rightarrow\left(H_{n}\right)_{0}$ such that $\delta(\kappa)=\delta(f \circ \theta)$ and $\kappa(0)=1$. Then $\kappa=$ $f \circ \theta$ by uniqueness of product integrals (cf. [19, Lemma 7.4]), entailing that $f(\theta([0,1]))=\kappa([0,1])$ is a compact subset of $\left(H_{n}\right)_{0}$. Note that if $y \in V$, then $Q \tau(y) \cap H_{0} \neq \emptyset$ if and only if $\tau(y) \in H_{0}$, in which case $Q \tau(y)$ is an open subset of $H_{0}$. Let

$$
D:=\left\{y \in V: Q \tau(y) \cap H_{0} \neq \emptyset\right\}
$$

Then $(Q \sigma(y))_{y \in D}$ is an open cover of $f(\theta([0,1]))$ by disjoint open subsets of $H_{0}$. Since $f(\theta([0,1]))$ is connected, it follows that $f(\theta([0,1])) \subseteq Q \sigma(0)=$ $Q$. Hence $f(q)=f(\theta(1)) \in Q$ in particular, establishing ([6) .

Because $P=Q \tau(V) \cong Q \times V$ and $Q \times\{0\}$ obviously is strongly $C^{k}$-initial in $Q \times V$, it follows that $Q$ is strongly $C^{k}$-initial in $P$. Since $f(Z) \subseteq Q$ and $\left.f\right|_{Z}$ is $C^{k}$ as a map to $P$, we infer that $\left.f\right|_{Z}: Z \rightarrow Q$ is $C^{k}$ and hence also $\left.f\right|_{Z}: Z \rightarrow H$.

It only remains to verify the claim. If $n=1$, there is one (and only one) choice of $\gamma_{1}, \eta_{1}:\{0\} \rightarrow\{1\}$. Now assume that $n \in \mathbb{N}$ and assume that $\gamma_{1}, \ldots, \gamma_{n}$ and $\eta_{1}, \ldots, \eta_{n}$ have already been constructed with the desired properties. Let $A \subseteq R_{n}$ be an open 0-neighbourhood and $\mu: A \rightarrow H_{n+1}$ be a smooth map such that $\mu(0)=1$ and $T_{0}(\mu)=\mathrm{id}_{R_{n}}$, identifying $T_{0}\left(R_{n}\right)$ with $R_{n}$. Consider the map

$$
\alpha: A \times U_{t_{n}}^{n} \times V_{t_{n}}^{n} \rightarrow G_{n+1}, \quad(a, x, y) \mapsto \mu(a) g_{n}(x, y)
$$

Then

$$
\operatorname{im}\left(T_{(0, x, y)}(\alpha)\right)=\gamma_{n}(x) \cdot\left(R_{n} \oplus L\left(G_{n}\right)\right) \cdot \eta_{n}(y)
$$

\footnotetext{
${ }^{5}$ Recall that if $\gamma:[0,1] \rightarrow G$ is $C^{1}$ then $\delta(\gamma)(t):=\gamma(t)^{-1} \cdot \gamma^{\prime}(t) \in L(G)$ defines $\delta(\gamma):[0,1] \rightarrow L(G)$ (cf. [19, p. 1043] and 21]). The dot denotes multiplication in the tangent group $T G$.
} 
for all $x \in U_{t_{n}}^{n}$ and $y \in V_{t_{n}}^{n}$ (where - denotes multiplication in the tangent group $\left.T G_{n+1}\right)$. In fact, abbreviating $c:=\gamma_{n}(x)$ and $e:=\eta_{n}(y)$, the image is

$$
\begin{aligned}
R_{n} \cdot c e+\operatorname{im}\left(T_{(x, y)} g_{n}\right) & =R_{n} \cdot c e+T_{c e} G_{n}=R_{n} \cdot c e+c \cdot L\left(G_{n}\right) \cdot e \\
& =R_{n} \cdot c e+c \cdot L\left(H_{n}\right) \cdot e+c \cdot E_{n} \cdot e \\
& =R_{n} \cdot c e+L\left(H_{n}\right) \cdot c e+c \cdot E_{n} \cdot e \\
& =\left(R_{n}+L\left(H_{n}\right)\right) \cdot c e+c \cdot E_{n} \cdot e \\
& =L\left(H_{n+1}\right) \cdot c e+c \cdot E_{n} \cdot e \\
& =c \cdot L\left(H_{n+1}\right) \cdot e+c \cdot E_{n} \cdot e \\
& =c \cdot\left(L\left(H_{n+1}\right)+E_{n}\right) \cdot e,
\end{aligned}
$$

where $L\left(H_{n+1}\right)+E_{n}=R_{n} \oplus L\left(H_{n}\right) \oplus E_{n}=R_{n} \oplus L\left(G_{n}\right)$.

We now equip $L\left(G_{n+1}\right)$ with an inner product, allowing us to consider orthogonal complements of vector subspaces of $L\left(G_{n+1}\right)$. Setting

$$
K_{y}:=\left(\eta_{n}(y)^{-1} \cdot\left(R_{n} \oplus L\left(G_{n}\right)\right) \cdot \eta_{n}(y)\right)^{\perp}
$$

for $y \in V_{t_{n}}^{n}$, we obtain a vector subbundle $K:=\bigcup_{y \in V_{t_{n}}^{n}}\{y\} \times K_{y}$ of the trivial bundle $V_{t_{n}}^{n} \times L\left(G_{n+1}\right) \rightarrow V_{t^{n}}^{n}$. Because $V_{t_{n}}^{n}$ is smoothly contractible, this vector bundle is trivial as a smooth vector bundle (by the $C^{\infty}$-version of [15, Chapter 2, Theorem 2.4]), and hence there exists a fibre-preserving $C^{\infty}$-diffeomorphism

$$
\phi: V_{t_{n}}^{n} \times S_{n} \rightarrow K
$$

where $V_{t_{n}}^{n} \times S_{n} \rightarrow V_{t_{n}}^{n}$ is the trivial bundle. Let $\pi_{2}: V_{t_{n}}^{n} \times L\left(G_{n+1}\right) \rightarrow L\left(G_{n+1}\right)$ be the projection onto the second factor and $\psi: C \rightarrow G_{n+1}$ be a $C^{\infty}$-map, defined on an open 0-neighbourhood $C \subseteq L\left(G_{n+1}\right)$, such that $\psi(0)=1$ and $T_{0} \psi=\operatorname{id}_{L\left(G_{n+1}\right)}$. Then $B:=\left(\pi_{2} \circ \phi\right)^{-1}(C)$ is an open neighbourhood of $V_{t_{n}}^{n} \times\{0\}$ in $V_{t_{n}}^{n} \times S_{n}$, and

$$
\nu: B \rightarrow G_{n+1}, \quad \nu(z):=\left(\psi \circ \pi_{2} \circ \phi\right)(z)
$$

is a smooth map such that $\nu(y, 0)=1$ for all $y \in V_{t_{n}}^{n}$ and

$$
\operatorname{im}\left(T_{(y, 0)} \nu\right)=\operatorname{im} T_{0} \nu(y, \bullet)=K_{y} .
$$

Then the smooth map

$$
\zeta: U_{t_{n}}^{n} \times A \times B \rightarrow G_{n+1}, \quad(x, a, y, b) \mapsto \mu(a) \gamma_{n}(x) \eta_{n}(y) \nu(y, b)
$$


(for $x \in U_{t_{n}}^{n}, a \in A, y \in V_{t_{n}}^{n}$ and $b \in S_{n}$ such that $(y, b) \in B$ ) satisfies

$$
\begin{aligned}
\operatorname{im}\left(T_{(x, 0, y, 0)} \zeta\right)= & \gamma_{n}(x) \cdot\left(R_{n} \oplus L\left(G_{n}\right)\right) \cdot \eta_{n}(y)+\gamma_{n}(x) \eta_{n}(y) \cdot K_{y} \\
= & \gamma_{n}(x) \eta_{n}(y) \cdot\left(\eta_{n}(y)^{-1} \cdot\left(R_{n} \oplus L\left(G_{n}\right)\right) \cdot \eta_{n}(y)\right. \\
& \left.\quad+\left(\eta_{n}(y)^{-1} \cdot\left(R_{n} \oplus L\left(G_{n}\right)\right) \cdot \eta_{n}(y)\right)^{\perp}\right) \\
= & \gamma_{n}(x) \eta_{n}(y) \cdot L\left(G_{n+1}\right)=T_{\zeta(x, 0, y, 0)} G_{n+1}
\end{aligned}
$$

using (7) for the first equality. Hence $T_{(x, 0, y, 0)} \zeta$ is a linear isomorphism (by reasons of dimension). The restriction of $\zeta$ to $\overline{U_{t_{n+1}}^{n}} \times\{0\} \times \overline{V_{t_{n+1}}^{n}} \times\{0\}$ corresponds to the restriction of $g_{n}$ to $\overline{U_{t_{n+1}}^{n}} \times \overline{V_{t_{n+1}}^{n}}$ and hence is injective. Thus Lemma 2.4 provides an open neighbourhood $\Omega$ of $\overline{U_{t_{n+1}}^{n}} \times\{0\} \times \overline{V_{t_{n+1}}^{n}} \times\{0\}$ in $U_{t_{n}}^{n} \times A \times B$ such that $\zeta(\Omega)$ is open in $G_{n+1}$ and $\left.\zeta\right|_{\Omega}$ is a $C^{\infty}$-diffeomorphism onto $\zeta(\Omega)$. There exists $r>0$ such that $U_{t_{n+1}}^{n} \times X_{r}^{n} \times V_{t_{n+1}}^{n} \times Y_{r}^{n} \subseteq \Omega$. Then

$$
\gamma_{n+1}: U_{t_{n+1}}^{n+1}=U_{t_{n+1}}^{n} \times X_{t_{n+1}}^{n} \rightarrow H_{n+1}, \quad \gamma_{n+1}(x, a):=\mu\left(\frac{r}{t_{n+1}} a\right) \gamma_{n}(x)
$$

and

$$
\eta_{n+1}: V_{t_{n+1}}^{n+1}=V_{t_{n+1}}^{n} \times Y_{t_{n+1}}^{n} \rightarrow G_{n+1}, \quad \eta_{n+1}(y, b):=\eta_{n}(y) \nu\left(y, \frac{r}{t_{n+1}} b\right)
$$

are smooth maps, and

$$
g_{n+1}: U_{t_{n+1}}^{n+1} \times V_{t_{n+1}}^{n+1} \rightarrow G_{n+1}, \quad g_{n+1}(x, y):=\gamma_{n+1}(x) \eta_{n+1}(y)
$$

is a $C^{\infty}$-diffeomorphism onto an open subset of $G_{n+1}$ (because so is $\left.\zeta\right|_{\Omega}$ ). Consequently, $\gamma_{n+1}$ is a $C^{\infty}$-diffeomorphism onto an open subset of $H_{n+1}$. By construction, $\gamma_{n+1}$ and $\eta_{n+1}$ also have all other required properties.

\section{Direct limit groups are topological groups with Lie algebra}

Given a (Hausdorff) topological group $G$, let $C(\mathbb{R}, G)$ be the set of continuous $G$-valued functions on $\mathbb{R}$, and $\operatorname{Hom}_{c}(\mathbb{R}, G)$ be the subset of all continuous homomorphisms from $(\mathbb{R},+)$ to $G$. We equip $C(\mathbb{R}, G)$ and $\operatorname{Hom}_{c}(\mathbb{R}, G)$ with the compact-open topology. Given $r \in \mathbb{R}$ and $\gamma \in \operatorname{Hom}_{c}(\mathbb{R}, G)$, we define $r \gamma \in \operatorname{Hom}_{c}(\mathbb{R}, G)$ via $(r \gamma)(t):=\gamma(t r)$ for $t \in \mathbb{R}$. Then

$$
\mathbb{R} \times \operatorname{Hom}_{c}(\mathbb{R}, G) \rightarrow \operatorname{Hom}_{c}(\mathbb{R}, G), \quad(r, \gamma) \mapsto r \gamma
$$

is a continuous map [16, p. 111-112]. We recall from [16, Definition 2.11]: 
Definition 3.1 $G$ is called a topological group with Lie algebra if

(a) $(\gamma+\eta)(t):=\lim _{n \rightarrow \infty}(\gamma(t / n) \eta(t / n))^{n}$ exists for all $\gamma, \eta \in \operatorname{Hom}_{c}(\mathbb{R}, G)$, and maps $\gamma+\eta: \mathbb{R} \rightarrow G$ so obtained are continuous homomorphisms;

(b) $\operatorname{Hom}_{c}(\mathbb{R}, G)$ is a topological vector space with the addition defined in (a) and the scalar multiplication (8); and

(c) There exists a continuous bilinear map

$$
[., .]: \operatorname{Hom}_{c}(\mathbb{R}, G) \times \operatorname{Hom}_{c}(\mathbb{R}, G) \rightarrow \operatorname{Hom}_{c}(\mathbb{R}, G)
$$

making it a Lie algebra, and such that

$$
[\gamma, \eta]\left(t^{2}\right)=\lim _{n \rightarrow \infty}(\gamma(t / n) \eta(t / n) \gamma(-t / n) \eta(-t / n))^{n^{2}},
$$

for all $\gamma, \eta \in \operatorname{Hom}_{c}(\mathbb{R}, G)$ and $t \in \mathbb{R}$.

It is known that a Lie group $G$ modelled on a locally convex space is a topological group with Lie algebra provided that $G$ is locally exponential in the sense that $G$ has a smooth exponential map $\exp _{G}: L(G) \rightarrow G$ which is a local diffeomorphism at 0 (see [21, Remark IV.1.22]). In particular, every Banach-Lie group and every finite-dimensional Lie group is a topological group with Lie algebra. Direct limits of finite-dimensional Lie groups always have a smooth exponential map, but they need not be locally exponential (see [5, Example 5.5]). Nonetheless, direct limit groups are topological groups with Lie algebra, as we verify now.

3.2 Recall that a Lie group $G$ is said to have an exponential map if, for each $x \in L(G)$, there exists a (necessarily unique) smooth homomorphism $\gamma_{x}: \mathbb{R} \rightarrow G$ with derivative $\gamma_{x}^{\prime}(0)=x$. In this case, define $\exp _{G}: L(G) \rightarrow G$, $\exp _{G}(x):=\gamma_{x}(1)$. Then $\gamma_{x}(t)=\exp _{G}(t x)$. As a first step towards our goal, consider a Lie group $G$ with the following properties:

(a) $G$ has an exponential map;

(b) Every continuous homomorphism $\mathbb{R} \rightarrow G$ is smooth;

(c) $\left(\gamma_{x}(t / n) \gamma_{y}(t / n)\right)^{n} \rightarrow \gamma_{x+y}(t)$ as $n \rightarrow \infty$ and

$$
\left(\gamma_{x}(t / n) \gamma_{y}(t / n) \gamma_{x}(-t / n) \gamma_{y}(-t / n)\right)^{n^{2}} \rightarrow \gamma_{[x, y]}\left(t^{2}\right),
$$

for all $x, y \in L(G)$ and $t \in \mathbb{R}$. 
Then the map

$$
\Gamma_{G}: L(G) \rightarrow \operatorname{Hom}_{c}(\mathbb{R}, G), \quad x \mapsto \gamma_{x}
$$

is a bijection. Furthermore, if we give $\operatorname{Hom}_{c}(\mathbb{R}, G)$ the unique Lie algebra structure which makes $\Gamma_{G}$ an isomorphism of Lie algebras, then conditions (a) and (c) from Definition 3.1 are satisfied, and the scalar multiplication of the Lie algebra $\operatorname{Hom}_{c}(\mathbb{R}, G)$ is given by (8) . Consequently, $G$ will be a topological group with Lie algebra if $\Gamma_{G}$ is a homeomorphism.

3.3 Now let $G_{1} \subseteq G_{2} \subseteq \cdots$ be finite-dimensional Lie groups, such that the inclusion maps $G_{n} \rightarrow G_{n+1}$ are smooth homomorphisms for all $n \in \mathbb{N}$. Let $G=\lim G_{n}$ be the direct limit Lie group. Then (a) and (c) hold (see [7. Proposition 4.6 (a) and (b)]), and also (b) (cf. [7, Proposition 4.6 (c)]). Moreover, the map $\Gamma_{G_{n}}: L\left(G_{n}\right) \rightarrow \operatorname{Hom}_{c}\left(\mathbb{R}, G_{n}\right.$ ) is a homeomorphism (cf. [21, Remark IV.1.22]). Since the inclusion map $\operatorname{Hom}_{c}\left(\mathbb{R}, G_{n}\right) \rightarrow \operatorname{Hom}_{c}(\mathbb{R}, G)$ is continuous and $L(G)=\lim _{\longrightarrow} L\left(G_{n}\right)$ as a topological space, $\Gamma_{G}$ is continuous.

Theorem 3.4 For each direct limit Lie group $G=\lim G_{n}$ as in $\mathbf{3 . 3}$, the mapping $\Gamma_{G}: L(G) \rightarrow \operatorname{Hom}_{c}(\mathbb{R}, G)$ is a homeomorphism and thus $G$ is a topological group with Lie algebra.

In view of the reduction steps performed in $\mathbf{3 . 2}$ and $\mathbf{3 . 3}$, it only remains to show that $\Gamma_{G}$ is an open map. This will follow from the next lemma.

3.5 It is useful to recall some standard facts first. Let $G$ be a topological group. By definition, the sets

$$
\lfloor K, U\rfloor_{G}:=\{\gamma \in C(\mathbb{R}, G): \gamma(K) \subseteq U\}
$$

form a subbasis of the compact-open topology on $C(\mathbb{R}, G)$, for $K$ ranging through the compact subsets of $\mathbb{R}$ and $U$ through the open subsets of $G$. It is known that $C(\mathbb{R}, G)$ is a topological group under pointwise multiplication: $(\gamma \cdot \eta)(t):=\gamma(t) \eta(t)$. As a consequence,

$$
\gamma \cdot\lfloor K, U\rfloor_{G}
$$

is an open neighbourhood of $\gamma$ for each $\gamma \in C(\mathbb{R}, G)$, compact set $K \subseteq \mathbb{R}$, and identity neighbourhood $U \subseteq G$. In fact, a basis of neighbourhoods of $\gamma$ is obtained in this way. Consequently, if $\gamma \in \operatorname{Hom}_{c}(\mathbb{R}, G)$, then

$$
\left(\gamma \cdot\lfloor K, U\rfloor_{G}\right) \cap \operatorname{Hom}_{c}(\mathbb{R}, G)
$$

is an open neighbourhood of $\gamma$ in $\operatorname{Hom}_{c}(\mathbb{R}, G)$, and the latter form a basis of neighbourhoods of $\gamma$. 
In the next lemma, $G_{1}$ and $G_{2}$ are finite-dimensional Lie groups such that $G_{1} \subseteq G_{2}$ and the inclusion map $G_{1} \rightarrow G_{2}$ is a smooth homomorphism. We identify $\mathfrak{g}_{1}:=L\left(G_{1}\right)$ with a Lie subalgebra of $\mathfrak{g}_{2}:=L\left(G_{2}\right)$ and assume that

$$
\left\{x \in \mathfrak{g}_{2}: \exp _{G_{2}}(\mathbb{R} x) \subseteq G_{1}\right\}=\mathfrak{g}_{1} .
$$

Lemma 3.6 Let $x \in \mathfrak{g}_{1}$ and $U_{j} \subseteq \mathfrak{g}_{j}$ be a relatively compact, open neighbourhood of $x$ for $j \in\{1,2\}$, such that $U_{1} \subseteq U_{2}$. Let $\varepsilon>0$ and $J:=[-\varepsilon, \varepsilon] \subseteq \mathbb{R}$. Let $V_{1} \subseteq G_{1}$ be a closed identity neighbourhood such that

$$
\left(\gamma_{x} \cdot\left\lfloor J, V_{1}\right\rfloor_{G_{1}}\right) \cap \operatorname{Hom}_{c}\left(\mathbb{R}, G_{1}\right) \subseteq \Gamma_{G_{1}}\left(U_{1}\right)
$$

and $R_{1}:=\left\{y \in \mathfrak{g}_{1}: \exp _{G_{1}}(J y) \subseteq V_{1}\right\}$ is compact. Then there exists a closed identity neighbourhood $V_{2} \subseteq G_{2}$ such that

$$
\left(\gamma_{x} \cdot\left\lfloor J, V_{2}\right\rfloor_{G_{2}}\right) \cap \operatorname{Hom}_{c}\left(\mathbb{R}, G_{2}\right) \subseteq \Gamma_{G_{2}}\left(U_{2}\right)
$$

and $V_{1}$ is contained in the interior $V_{2}^{0}$ of $V_{2}$ relative $G_{2}$. Furthermore, one can achieve that

$$
R_{2}:=\left\{y \in \mathfrak{g}_{2}: \exp _{G_{2}}(J y) \subseteq V_{2}\right\}
$$

is compact. If $V_{1}$ is compact, then also $V_{2}$ can be chosen compact.

Proof. Let $Q_{1} \supseteq Q_{2} \supseteq \cdots$ be a basis of compact identity neighbourhoods in $G_{2}$, and

$$
W_{m}:=V_{1} Q_{m} \quad \text { for } m \in \mathbb{N} .
$$

Then (11) holds with $V_{2}:=W_{m}$ for all sufficiently large $m \in \mathbb{N}$. In fact, otherwise we could find positive integers $m_{1}<m_{2}<\cdots$ and elements $\eta_{n} \in$ $\left\lfloor J, W_{m_{n}}\right\rfloor$ for $n \in \mathbb{N}$ such that

$$
\zeta_{n}:=\gamma_{x} \cdot \eta_{n} \in \operatorname{Hom}_{c}\left(\mathbb{R}, G_{2}\right)
$$

and $\zeta_{n} \notin \Gamma_{G_{2}}\left(U_{2}\right)$. Then $\zeta_{n}=\Gamma_{G_{2}}\left(z_{n}\right)$ with $z_{n}:=\Gamma_{G_{2}}^{-1}\left(\zeta_{n}\right) \in \mathfrak{g}_{2} \backslash U_{2}$.

Case 1: If $\left(z_{n}\right)_{n \in \mathbb{N}}$ has a convergent subsequence, then we may assume (after passage to the latter) that $z_{n} \rightarrow z$ as $n \rightarrow \infty$ for some $z \in \mathfrak{g}_{2}$. Define $\eta:=\gamma_{x}^{-1} \cdot \gamma_{z} \in C\left(\mathbb{R}, G_{2}\right)$. Then

$$
\eta(J) \subseteq V_{1}
$$


In fact, for each $t \in J$ and $n \in \mathbb{N}$, we have

$$
\begin{aligned}
\eta(t) & =\gamma_{x}(t)^{-1} \exp _{G_{2}}(t z)=\lim _{k \rightarrow \infty} \gamma_{x}(t)^{-1} \exp _{G_{2}}\left(t z_{k}\right) \\
& =\lim _{k \rightarrow \infty} \gamma_{x}(t)^{-1} \zeta_{k}(t)=\lim _{k \rightarrow \infty} \eta_{k}(t) \in W_{m_{n}}
\end{aligned}
$$

because $\eta_{k}(t) \in W_{m_{k}} \subseteq W_{m_{n}}$ and $W_{m_{n}}$ is closed. Hence

$$
\eta(t) \in \bigcap_{n \in \mathbb{N}} W_{m_{n}}=V_{1}
$$

as asserted (where the last equality holds by [24, Lemma 3.17]).

As a consequence of (12), we have $\gamma_{z}(t)=\gamma_{x}(t) \eta(t) \in G_{1}$ for each $t \in J$ and hence $\exp _{G_{2}}(\mathbb{R} z)=\gamma_{z}(\mathbb{R}) \subseteq G_{1}$, whence $z \in \mathfrak{g}_{1}$, by (9). Hence $\gamma_{z} \in$ $\operatorname{Hom}_{c}\left(\mathbb{R}, G_{1}\right)$ and thus $\eta \in C\left(\mathbb{R}, G_{1}\right)$. By (12), we have $\eta \in\left\lfloor J, V_{1}\right\rfloor_{G_{1}}$. Hence

$$
\gamma_{z}=\gamma_{x} \eta \in\left(\gamma_{x}\left\lfloor J, V_{1}\right\rfloor_{G_{1}}\right) \cap \operatorname{Hom}_{c}\left(\mathbb{R}, G_{1}\right)
$$

whence $\gamma_{z} \in \Gamma_{G_{1}}\left(U_{1}\right)$ (by (10)) and thus $z \in U_{1}$. But $z \in \mathfrak{g}_{2} \backslash U_{2}$ and hence $z \notin U_{1}$, contradiction.

Case 2: If $\left(z_{n}\right)_{n \in \mathbb{N}}$ has no convergent subsequence, after passing to a subsequence we may assume that $\left\|z_{n}\right\| \rightarrow \infty$ as $n \rightarrow \infty$, where $\|$.$\| is a given$ norm on $\mathfrak{g}_{2}$. Since $R_{1}$ is compact, there exists $r>0$ such that

$$
R_{1} \subseteq\left\{y \in \mathfrak{g}_{1}:\|y\|<r\right\}=: B_{1} .
$$

We let $B_{2}:=\left\{y \in \mathfrak{g}_{2}:\|y\|<2 r\right\}$. As $2 r z_{n} /\left\|z_{n}\right\|$ is contained in the compact set $\partial B_{2}$, after passage to a subsequence we may assume that $2 r z_{n} /\left\|z_{n}\right\| \rightarrow b$ as $n \rightarrow \infty$ for some $b \in \partial B_{2}$. Abbreviate $r_{n}:=2 r /\left\|z_{n}\right\|$. Then $r_{n} \rightarrow 0$, and we may assume that $r_{n} \leq 1$ for each $n$. For each $t \in J$, we then have

$$
\eta_{n}\left(t r_{n}\right)=\exp _{G_{2}}\left(-t r_{n} x\right) \exp _{G_{2}}\left(t r_{n} z_{n}\right) \rightarrow \exp _{G_{2}}(t b) \text { as } n \rightarrow \infty \text {. }
$$

Since $\eta_{n}\left(t r_{n}\right) \in W_{m_{n}}$, it follows that $\exp _{G_{2}}(t b) \in \bigcap_{n \in \mathbb{N}} W_{m_{n}}=V_{1}$. Hence $\exp _{G_{2}}(\mathbb{R} b) \subseteq G_{1}$ and thus $b \in \mathfrak{g}_{1}$. By the preceding, we have $b \in R_{1}$ and therefore $b \in B_{1}$, by (13). But $b \in \partial B_{2}$, whence $b \notin B_{2}$ and thus $b \notin B_{1}$, contradiction.

The final assertion is clear, since compactness of $V_{1}$ entails that also each $W_{m}$ is compact. It only remains to show that $R_{2}$ can be chosen compact. 
However, applying the results already shown with $1, B_{1}$ and $B_{2}$ instead of $x$, $U_{1}$ and $U_{2}$, respectively, we see that

$$
\left\{y \in \mathfrak{g}_{2}: \exp _{G_{2}}(J y) \subseteq W_{m}\right\} \subseteq B_{2}
$$

for all sufficiently large $m$. For such $m$, the set on the left is compact.

Proof of Theorem 3.4. Since $G_{0}=\lim \left(G_{n}\right)_{0}$ (see 2.2), $L(G)=L\left(G_{0}\right)$, $\operatorname{Hom}_{c}(\mathbb{R}, G)=\operatorname{Hom}_{c}\left(\mathbb{R}, G_{0}\right)$ and $\Gamma_{G}=\vec{\Gamma}_{G_{0}}$, after replacing $G$ with $G_{0}$ and each $G_{n}$ with $G_{n, 0}$ we may assume that $G$ and each $G_{n}$ is connected. The discussion leading to (1) now shows that

$$
L\left(G_{n}\right)=\left\{x \in L\left(G_{n+1}\right): \exp _{G_{n+1}}(\mathbb{R} x) \subseteq G_{n}\right\}
$$

(as needed in Lemma 3.6).

It remains to show that $\Gamma_{G}(U)$ is open in $\operatorname{Hom}_{c}(\mathbb{R}, G)$ for each open subset $U \subseteq L(G)=\bigcup_{n \in \mathbb{N}} L\left(G_{n}\right)$. We verify that $\Gamma_{G}(U)$ is a neighbourhood of $\Gamma_{G}(x)$ for each $x \in U$. After passage to a cofinal subsequence, we may assume that $x \in L\left(G_{1}\right)$. Then $U_{n}:=L\left(G_{n}\right) \cap U$ is an open neighbourhood of $x$ in $L\left(G_{n}\right)$ for each $n \in \mathbb{N}$, and $U_{1} \subseteq U_{2} \subseteq \cdots$. Since $\Gamma_{G_{1}}\left(U_{1}\right)$ is an open neighbourhood of $\gamma_{x}$ in $\operatorname{Hom}_{c}\left(\mathbb{R}, G_{1}\right)$, there exists $\varepsilon>0$ and an open identity neighbourhood $P \subseteq G_{1}$ such that

$$
\left(\gamma_{x}\lfloor J, P\rfloor_{G_{1}}\right) \cap \operatorname{Hom}_{c}\left(\mathbb{R}, G_{1}\right) \subseteq \Gamma_{G_{1}}\left(U_{1}\right),
$$

where $J:=[-\varepsilon, \varepsilon]$ (cf. 3.5). There exists a compact identity neighbourhood $V_{1} \subseteq P$ such that

$$
R_{1}:=\left\{y \in L\left(G_{1}\right): \exp _{G_{1}}(J y) \subseteq V_{1}\right\}
$$

is compact. Using now Lemma 3.6 and induction, we find compact identity neighbourhoods $V_{n} \subseteq G_{n}$ for all integers $n \geq 2$ such that

(i) $V_{n-1} \subseteq V_{n}^{0}$ (the interior relative $G_{n}$ );

(ii) $\left(\gamma_{x} \cdot\left\lfloor J, V_{n}\right\rfloor_{G_{n}}\right) \cap \operatorname{Hom}_{c}\left(\mathbb{R}, G_{n}\right) \subseteq \Gamma_{G_{n}}\left(U_{n}\right)$, and

(iii) $R_{n}:=\left\{y \in L\left(G_{n}\right): \exp _{G_{n}}(J y) \subseteq V_{n}\right\}$ is compact. 
Since $Q_{n}:=V_{n}^{0}$ is open in $G_{n}$ and $Q_{1} \subseteq Q_{2} \subseteq \cdots$, the set $Q:=\bigcup_{n \in \mathbb{N}} Q_{n}$ is open in $G$ (see 2.3). Then

$$
\left(\gamma_{x} \cdot\lfloor J, Q\rfloor_{G}\right) \cap \operatorname{Hom}_{c}(\mathbb{R}, G)
$$

is an open neighbourhood of $\gamma_{x}$ in $\operatorname{Hom}_{c}(\mathbb{R}, G)$. The proof will be complete if we can show that

$$
\left(\gamma_{x} \cdot\lfloor J, Q\rfloor_{G}\right) \cap \operatorname{Hom}_{c}(\mathbb{R}, G) \subseteq \Gamma_{G}(U) .
$$

To verify (14), let $\eta \in\lfloor J, Q\rfloor_{G}$ such that $\zeta:=\gamma_{x} \cdot \eta \in \operatorname{Hom}_{c}(\mathbb{R}, G)$. Then $\zeta=\gamma_{z}$ with $z:=\Gamma_{G}^{-1}(\zeta)$. There is $n \in \mathbb{N}$ such that $z \in L\left(G_{n}\right)$ and thus $\zeta \in \operatorname{Hom}_{c}\left(\mathbb{R}, G_{n}\right)$. Then $\eta=\gamma_{x}^{-1} \cdot \zeta \in C\left(\mathbb{R}, G_{n}\right)$. Since $\eta(J) \subseteq G_{n}$ is compact and $\left(Q_{m} \cap G_{n}\right)_{m \geq n}$ is an open cover of $\eta(J)$, there exists $m \geq n$ such that $\eta(J) \subseteq Q_{m}$. Thus $\eta \in\left\lfloor J, Q_{m}\right\rfloor_{G_{m}} \subseteq\left\lfloor J, V_{m}\right\rfloor_{G_{m}}$ and hence

$$
\zeta=\gamma_{x} \cdot \eta \in\left(\gamma_{x} \cdot\left\lfloor J, V_{m}\right\rfloor_{G_{m}}\right) \cap \operatorname{Hom}_{c}\left(\mathbb{R}, G_{m}\right) \subseteq \Gamma_{G_{m}}\left(U_{m}\right),
$$

using (ii). Then $\zeta \in \Gamma_{G_{m}}\left(U_{m}\right) \subseteq \Gamma_{G}(U)$ and thus (14) is established.

If a Lie group is a topological group with Lie algebra, then this has useful consequences. We recall that it is an unsolved open problem (first formulated by John Milnor) whether every continuous homomorphism between infinitedimensional Lie groups is smooth (as in the finite-dimensional case). The following result provides some positive information.

Proposition 3.7 Let $H$ be a locally exponential Lie group and $G$ be a Lie group such that (a)-(c) from $\mathbf{3 . 2}$ are satisfied, $\Gamma_{G}: L(G) \rightarrow \operatorname{Hom}_{c}(\mathbb{R}, G)$ is a homeomorphism, and $\exp _{G}: L(G) \rightarrow G$ is smooth. Then every continuous homomorphism $\alpha: H \rightarrow G$ is smooth.

Proof. The map $\beta:=\operatorname{Hom}_{c}(\mathbb{R}, \alpha): \operatorname{Hom}_{c}(\mathbb{R}, H) \rightarrow \operatorname{Hom}_{c}(\mathbb{R}, G), \gamma \mapsto \alpha \circ \gamma$ is continuous. Since

$$
\begin{aligned}
\beta(\gamma+\eta)(t) & =\alpha\left(\lim _{n \rightarrow \infty}(\gamma(t / n) \eta(t / n))^{n}\right) \\
& =\lim _{n \rightarrow \infty}((\alpha \circ \gamma)(t / n)(\alpha \circ \eta)(t / n))^{n}=(\beta(\gamma)+\beta(\eta))(t)
\end{aligned}
$$

for all $\gamma, \eta \in \operatorname{Hom}_{c}(\mathbb{R}, H)$, we see that $\beta$ is a homomorphism of groups and hence a continuous linear map. Then $\theta:=\Gamma_{G}^{-1} \circ \beta \circ \Gamma_{H}: L(H) \rightarrow L(G)$ is a continuous linear (and hence smooth) map such that $\exp _{G} \circ \theta=\alpha \circ \exp _{H}$. Since $\exp _{G} \circ \theta$ is smooth and $\exp _{H}$ is a local diffeomorphism, it follows that $\alpha$ is smooth on some open identity neighbourhood and hence smooth. 


\section{Borel-type theorem for diffeomorphisms}

This section is devoted to the proof of Borel's Theorem for Diffeomorphisms, as stated in the introduction.

As usual, $\mathrm{GL}_{n}(\mathbb{R})$ denotes the group of all invertible $n \times n$-matrices and $\operatorname{GL}\left(\mathbb{R}^{n}\right)$ the group of all automorphisms of the real vector space $\mathbb{R}^{n}$. The algebra of all linear endomorphism of $\mathbb{R}^{n}$ will be denoted by $\mathcal{L}\left(\mathbb{R}^{n}\right)$. If $M$ is a finite-dimensional smooth manifold and $K \subseteq M$, we let $\operatorname{Diff}_{K}(M)$ be the Lie group of all $C^{\infty}$-diffeomorphisms $\gamma: M \rightarrow M$ such that $\gamma(x)=x$ for all $x \in M \backslash K$ (see, e.g., [9]; cf. [18]). We write $C_{K}^{\infty}(M)$ for the space of smooth maps $\gamma: M \rightarrow \mathbb{R}$ such that $\left.\gamma\right|_{M \backslash K}=0$. Finally, we let $C^{\infty}\left(\mathbb{R}^{n}, \mathbb{R}^{n}\right)$ be the space of $\mathbb{R}^{n}$-valued smooth maps on $\mathbb{R}^{n}$, equipped with the usual locally convex topology (the smooth compact-open topology).

Lemma 4.1 Let $n \in \mathbb{N}, A \in \mathrm{GL}\left(\mathbb{R}^{n}\right)$ such that $\operatorname{det}(A)>0$, and $K \subseteq \mathbb{R}^{n}$ be a compact 0-neighbourhood. Then there exists a smooth map

$$
\zeta: \mathbb{R} \rightarrow \operatorname{Diff}_{K}\left(\mathbb{R}^{n}\right)
$$

with $\zeta(0)=\operatorname{id}_{\mathbb{R}^{n}}$, such that

$$
\theta:=\zeta(1)
$$

satisfies $\left.\theta\right|_{U}=\left.A\right|_{U}$ for some 0-neighbourhood $U \subseteq K$.

Proof. Let $R$ be a compact 0-neighbourhood contained in the interior $K^{0}$ of $K$. Then there is $\chi \in C_{K}^{\infty}\left(\mathbb{R}^{n}\right)$ such that $\left.\chi\right|_{R}=1$. It is well known that

$$
\left\{B \in \mathrm{GL}\left(\mathbb{R}^{n}\right): \operatorname{det}(B)>0\right\}
$$

is the connected component of $\mathrm{GL}\left(\mathbb{R}^{n}\right)$. We therefore find a smooth map $f: \mathbb{R} \rightarrow \mathrm{GL}\left(\mathbb{R}^{n}\right)$ with $f(0)=\operatorname{id}_{\mathbb{R}^{n}}$ and $f(1)=A$. Let $f^{\prime}(t) \in \mathcal{L}\left(\mathbb{R}^{n}\right)$ be the derivative of $f$ at $t \in \mathbb{R}$. We consider the time-dependent smooth vector field

$$
v: \mathbb{R} \times \mathbb{R}^{n} \rightarrow \mathbb{R}^{n}, \quad v(t, x):=\left(f^{\prime}(t) \circ f(t)^{-1}\right)(x)
$$

on $\mathbb{R}^{n}$. For each $x \in \mathbb{R}^{n}$,

$$
\gamma_{x}: \mathbb{R} \rightarrow \mathbb{R}^{n}, \quad \gamma_{x}(t):=f(t)(x)
$$

is a smooth curve such that $\gamma_{x}(0)=x$ and

$$
\gamma_{x}^{\prime}(t)=f^{\prime}(t)(x)=\left(f^{\prime}(t) \circ f(t)^{-1}\right)(f(t)(x))=v\left(t, \gamma_{x}(t)\right) .
$$


Thus $\gamma_{x}$ solves the initial value problem

$$
y^{\prime}(t)=v(t, y(t)), \quad y(0)=x
$$

Hence $\Phi_{v}: \mathbb{R} \times \mathbb{R}^{n} \rightarrow \mathbb{R}^{n}, \Phi_{v, t}(x):=\gamma_{x}(t)$ is the flow of $v$ (for fixed initial time $\left.t_{0}=0\right)$. Now consider

$$
w: \mathbb{R} \times \mathbb{R}^{n} \rightarrow \mathbb{R}^{n}, \quad w(t, x):=\chi(x) v(t, x) .
$$

Then $w$ admits a smooth flow $\Phi_{w}: \mathbb{R} \times \mathbb{R}^{n} \rightarrow \mathbb{R}^{n}$, and $\zeta(t):=\Phi_{w, t} \in$ $\operatorname{Diff}_{K}\left(\mathbb{R}^{n}\right)$ for each $t \in \mathbb{R}$. Since $\phi_{w, t}(0)=0$ for each $t \in \mathbb{R}$ and the flow is continuous, we find a 0 -neighbourhood $U \subseteq K$ such that

$$
\Phi_{w, t}(x) \in R^{0} \quad \text { for all } x \in U \text { and } t \in[0,1]
$$

Since $v$ and $w$ coincide on $\mathbb{R} \times R^{0}$, it follows that $\Phi_{w, t}(x)=\Phi_{v, t}(x)$ for all $x \in U$ and $t \in[0,1]$, entailing that

$$
\Phi_{w, 1}(x)=\Phi_{v, 1}(x)=\gamma_{x}(1)=A(x) \quad \text { for all } x \in U
$$

By construction of the Lie group structure on $\operatorname{Diff}_{K}\left(\mathbb{R}^{n}\right)$, the map

$$
\kappa: \operatorname{Diff}_{K}\left(\mathbb{R}^{n}\right) \rightarrow C_{K}^{\infty}\left(\mathbb{R}^{n}, \mathbb{R}^{n}\right), \quad \gamma \mapsto \gamma-\mathrm{id}_{\mathbb{R}^{n}}
$$

is a diffeomorphism onto an open subset of $C_{K}^{\infty}\left(\mathbb{R}^{n}, \mathbb{R}^{n}\right)$ (cf. [6]). Because

$$
\mathbb{R} \times \mathbb{R}^{n} \rightarrow \mathbb{R}^{n}, \quad(t, x) \mapsto\left(\zeta(t)-\mathrm{id}_{\mathbb{R}^{n}}\right)(x)=\Phi_{w, t}(x)-x
$$

is a smooth mapping, the exponential law for smooth mappings (see, e.g., [10, Lemma $12.1(\mathrm{a})]$ ) entails that $\kappa \circ \zeta$ (and hence also $\zeta$ ) is smooth. Since $\zeta(0)=\operatorname{id}_{\mathbb{R}^{n}}$ and $\zeta(1)=\Phi_{w, 1}$, we see that $\zeta$ has all required properties.

Given $n \in \mathbb{N}$ and $j \in\{1, \ldots, n\}$, let $e_{j}:=(0, \ldots, 0,1,0, \ldots, 0)^{T} \in \mathbb{R}^{n}$ with 1 in the $j$-th slot.

Lemma 4.2 Let $n \in \mathbb{N}$ and $a_{\alpha} \in \mathbb{R}^{n}$ for multi-indices $\alpha \in \mathbb{N}_{0}^{n}$ be given such that $A:=\left(a_{e_{1}}, \ldots, a_{e_{n}}\right) \in \mathrm{GL}_{n}(\mathbb{R})$ and $\operatorname{det}(A)>0$. Then there exists $\phi \in \operatorname{Diff}_{c}\left(\mathbb{R}^{n}\right)$ such that $\frac{\left(\partial^{\alpha} \phi\right)(0)}{\alpha !}=a_{\alpha}$ for each $\alpha \in \mathbb{N}_{0}^{n}$. 
Proof. After a translation, we may assume that $a_{0}=0$. Then the vectors $a_{\alpha}$ determine a formal diffeomorphism $f=\sum_{\alpha} a_{\alpha} x^{\alpha}$ of $\mathbb{R}^{n}$. Considering $A$ as a formal diffeomorphism, the formal composition $g:=A^{-1} \circ f$ is of the form $g=\sum_{\alpha} b_{\alpha} x^{\alpha}$ with vectors $b_{\alpha} \in \mathbb{R}^{n}$ such that $\left(b_{e_{1}}, \ldots, b_{e_{n}}\right)=\mathbf{1}$ is the identity matrix. Let $\theta$ be as in the previous lemma. If we can prove the lemma with the $b_{\alpha}$ in place of the $a_{\alpha}$, leading to $\phi$, then $\kappa:=\theta \circ \phi$ is a diffeomorphism such that $\left.\kappa\right|_{W}=\left.A \circ \phi\right|_{W}$ for some 0-neighbourhood $W \subseteq \mathbb{R}^{n}$, and hence $\frac{\left(\partial^{\alpha} \kappa\right)(0)}{\alpha !}=a_{\alpha}$ for each $\alpha \in \mathbb{N}_{0}^{n}$. We may therefore assume now that $A=\mathbf{1}$.

Let $\|$.$\| be the maximum norm on \mathbb{R}^{n}$. Then $\left\{x \in \mathbb{R}^{n}:\|x\| \leq \varepsilon\right\} \subseteq K$ for some $\varepsilon>0$. Let $h: \mathbb{R} \rightarrow \mathbb{R}$ be a compactly supported smooth function such that $h(x)=x$ for $x$ in some 0-neighbourhood, and $h(x)=0$ if $|x| \geq \varepsilon$. Define $h_{\alpha}\left(x_{1}, \ldots, x_{n}\right):=h\left(x_{1}\right)^{\alpha_{1}} \cdots h\left(x_{n}\right)^{\alpha_{n}}$ and

$$
M_{k, m}:=\max _{|\beta|=m} \sum_{|\alpha|=k}\left\|a_{\alpha}\right\| \cdot\left\|\partial^{\beta} h_{\alpha}\right\|_{\infty}
$$

where $\|\cdot\|_{\infty}$ is the supremum norm on the space $C_{0}(\mathbb{R})$ real-valued functions that vanish at infinity, and $|\alpha|=\alpha_{1}+\cdots+\alpha_{n}$ the order of the multi-index $\alpha \in \mathbb{N}_{0}^{n}$. For each $k \in \mathbb{N}$ with $k \geq 2$, pick $c_{k}>1$ such that

$$
c_{k}^{m-k} M_{k, m}<\frac{2^{-k}}{n}
$$

for all $m \in \mathbb{N}_{0}$ such that $m<k$. Define $\phi_{k}: \mathbb{R}^{n} \rightarrow \mathbb{R}^{n}$ via $\phi_{k}(x):=$ $\sum_{|\alpha|=k} a_{\alpha} c_{k}^{-k} h_{\alpha}\left(c_{k} x\right)$. Then $\phi_{k}$ is smooth and

$$
\partial^{\beta} \phi_{k}(x)=c_{k}^{|\beta|-k} \sum_{|\alpha|=k} a_{\alpha}\left(\partial^{\beta} h_{\alpha}\right)\left(c_{k} x\right),
$$

whence

$$
\left\|\partial^{\beta} \phi_{k}\right\|_{\infty} \leq c_{k}^{|\beta|-k} M_{k,|\beta|},
$$

which is $\leq \frac{2^{-k}}{n}$ for all $k \in \mathbb{N}$ such that $k>|\beta|$. Hence the limit $\psi:=$ $\sum_{k=2}^{\infty} \phi_{k}$ exists in $C^{\infty}\left(\mathbb{R}^{n}, \mathbb{R}^{n}\right)$, and clearly $\psi$ has compact support. By the preceding, for $|\beta|=1$ we have $\left\|\partial^{\beta} \phi_{k}\right\|_{\infty} \leq \frac{2^{-k}}{n}$ for each $k \geq 2$ and thus $\left\|\partial^{\beta} \psi\right\|_{\infty} \leq \sum_{k=2}^{\infty} \frac{2^{-k}}{n}=\frac{1}{2 n}$, whence $\left\|\psi^{\prime}(x)\right\|_{\text {op }} \leq 1 / 2$ for each $x \in \mathbb{R}^{n}$ and thus $\sup \left\{\left\|\psi^{\prime}(x)\right\|_{\text {op }}: x \in \mathbb{R}^{n}\right\} \leq 1 / 2<1$. Therefore $\phi:=\operatorname{id}_{\mathbb{R}^{n}}+\psi \in \operatorname{Diff}_{c}\left(\mathbb{R}^{n}\right)$ (see [6, Lemma 5.1]). By construction, $\frac{\partial^{\alpha} \phi(0)}{\alpha !}=a_{\alpha}$ for each $\alpha$.

Proof of Borel's Theorem for Diffeomorphisms. Let $p$ and a chart $\phi: M \supseteq U \rightarrow V \subseteq \mathbb{R}^{n}$ be as described in the theorem. After shrinking $U$, we 
may assume that $V$ is a euclidean ball with center 0 . Composing now with a diffeomorphism $V \rightarrow \mathbb{R}^{n}$ which is the identity on some 0-neighbourhood and fixes 0 , we can (and shall) assume instead that $V=\mathbb{R}^{n}$. Let $C \subseteq U$ be a compact neighbourhood of $p$ and $K:=\phi(C)$. Then the restriction map

$$
\operatorname{Difff}_{C}(M) \rightarrow \operatorname{Diff}_{C}(U)
$$

is an isomorphism of Lie groups and so is the map

$$
\operatorname{Diff}_{C}(M) \rightarrow \operatorname{Diff}_{K}\left(\mathbb{R}^{n}\right), \quad \gamma \mapsto \phi \circ \gamma \circ \phi^{-1}
$$

(this is clear from the construction of the Lie group structure in [9]). It therefore suffices to consider the case where $M=\mathbb{R}^{n}, \phi=\mathrm{id}_{\mathbb{R}^{n}}$ and $p=0$. In this case, the surjectivity of

$$
T_{p}^{\infty}: \operatorname{Diff}_{c}\left(\mathbb{R}^{n}\right)_{p, 0} \rightarrow \mathrm{Gf}_{n}(\mathbb{R})_{0}
$$

has been established in Lemma 4.2, The Chain Rule for Taylor Polynomials entails that $T_{p}^{\infty}$ is a homomorphism of groups. Since $\mathrm{Gf}_{n}(\mathbb{R})_{0}$ is an open subset of $\left\{\sum_{\alpha} a_{\alpha} x^{\alpha} \in \mathbb{R} \llbracket x_{1}, \ldots, x_{n} \rrbracket: a_{0}=0\right\} \cong \mathbb{R}^{\mathbb{N}_{0}^{n} \backslash\{0\}}$ with the product topology, and each component

$$
\left(T_{p}^{\infty}\right)_{\alpha}: \operatorname{Diff}_{c}\left(\mathbb{R}^{n}\right)_{p, 0} \rightarrow \mathbb{R}, \quad \gamma \mapsto \frac{\left(\partial^{\alpha} \gamma\right)(0)}{\alpha !}
$$

is smooth, we deduce that $T_{p}^{\infty}$ is smooth. This completes the proof.

Acknowledgements. The author thanks K.-H. Neeb for communicating early versions of his survey, and for comments on a draft of the current article.

\section{References}

[1] Bertram, W., H. Glöckner and K.-H. Neeb, Differential calculus over general base fields and rings, Expo. Math. 22 (2004), 213-282.

[2] Bourbaki, N., "Lie Groups and Lie Algebras, Chapters 1-3," SpringerVerlag, 1989.

[3] Dahmen, R. and H. Glöckner, Regularity in Milnor's sense for direct limits of infinite-dimensional Lie groups, in preparation. 
[4] Glöckner, H., Infinite-dimensional Lie groups without completeness restrictions, pp. 43-59 in: A. Strasburger et al. (eds.) "Geometry and analysis on finite- and infinite-dimensional Lie groups," Banach Center Publ. 55 (2002), 43-59.

[5] Glöckner, H., Direct limit Lie groups and manifolds, J. Math. Kyoto Univ. 43 (2003), 1-26.

[6] Glöckner, H., Diff $\left(\mathbb{R}^{n}\right)$ as a Milnor-Lie group, Math. Nachr. 278 (2005), 1025-1032.

[7] Glöckner, H., Fundamentals of direct limit Lie theory, Compos. Math. 141 (2005), 1551-1577.

[8] Glöckner, H., Direct limits of infinite-dimensional Lie groups compared to direct limits in related categories, J. Funct. Anal. 245 (2007), 19-61.

[9] Glöckner, H., Patched locally convex spaces, almost local mappings and diffeomorphism groups of non-compact manifolds, manuscript, 2002.

[10] Glöckner, H., Lie groups over non-discrete topological fields, preprint, arXiv:math/0408008v1.

[11] Glöckner, H. and L.R. Lovas, Frobenius and Stefan-Sussmann theorems for various types of distributions on infinite-dimensional manifolds, manuscript in preparation.

[12] Glöckner, H. and K.-H. Neeb, "Infinite-Dimensional Lie Groups, Vol. I" book in preparation.

[13] Hiltunen, S., A Frobenius theorem for locally convex global analysis, Monatsh. Math. 129 (2000), 109-117.

[14] Hirai, T., H. Shimomura, N. Tatsuuma and E. Hirai, Inductive limits of topologies, their direct product, and problems related to algebraic structures, J. Math. Kyoto Univ. 41 (2001), 475-505.

[15] Hirsch, M. W., "Differential Topology," Springer-Verlag, 1976.

[16] Hofmann, K. H. and S. A. Morris, "The Structure of Connected Pro-Lie Groups," EMS Tracts in Math. 2, EMS Publ. House, Zurich, 2007. 
[17] Kriegl, A. and P. W. Michor, "The Convenient Setting of Global Analysis," Amer. Math. Soc., Providence, 1997.

[18] Michor, P. W., "Manifolds of differentiable mappings," Shiva Publishing, Nantwich, 1980.

[19] Milnor, J., Remarks on infinite-dimensional Lie groups. pp. 1007-1057 in: Relativity, groups and topology, II (Les Houches, 1983), NorthHolland, Amsterdam, 1984.

[20] Natarajan, L., E. Rodríguez-Carrington and J.A. Wolf, Differentiable structure for direct limit groups, Letters in Math. Phys. 23 (1991), 99109.

[21] Neeb, K.-H., Towards a Lie theory of locally convex groups, Jpn. J. Math. 1 (2006), 291-468.

[22] Pisanelli, D., An example of an infinite Lie group, Proc. Amer. Math. Soc. 62 (1977), 156-160.

[23] Robart, T. and N. Kamran, Sur la théorie locale des pseudogroupes de transformations continus infinis I, Math. Ann. 308 (1997), 593-613.

[24] Stroppel, M., "Locally Compact Groups," EMS Publ. House, Zurich, 2006.

Helge Glöckner, Universität Paderborn, Institut für Mathematik, Warburger Str. 100, 33098 Paderborn, Germany. E-Mail: glockner@math.uni-paderborn.de 